Pre-print: Dorin, A., "Enriching Aesthetics with Artificial Life", in Artificial Life Models In Software, Komosinski and Adamatzky (eds), Springer-Verlag, 2005, pp323-335, ISBN 1-85233-945-4

\title{
Enriching Aesthetics with Artificial Life
}

\author{
Alan Dorin \\ Center for Electronic Media Art, \\ School of Computer Science and Software Engineering, \\ Monash University, \\ Australia 3800 \\ aland@csse.monash.edu.au
}

Perhaps one day our machines will be able to take us beyond environments which look more and more familiar as we traverse them, into spaces which continue to surprise us as we explore. Can our engineered artefacts ever rival the intricacy of nature?

\section{Introduction.}

Artificial Life is studied largely as a means of furthering our understanding of biology and of complex adaptive systems in general. Whilst it has demonstrated potential in a number of fields, in particular as a means of solving engineering problems, Artificial Life techniques have also been applied by some in the art community. The field promises to continue to enrich artistic practice and our approach to contemporary aesthetics, even as its initial flash of popularity wanes. It is this continued application to aesthetics which the present paper begins to address.

The techniques of art based in Artificial Life form a subset of generative art. This is an artistic practice which adopts an aesthetic of process. By this it is meant that although the final outcome of a work may depend for its appeal on the aesthetics of an image, sound, sculpture or other form, the process which generates it is also significant. The generative artist is responsible for setting up initial conditions and a process to act upon them. The work which unfolds is the result of this series of changes. This is analogous to a biological phenotype (usually an organism and its behaviour) being the result of the physical and chemical interactions which govern its development from a genotype (its genetic material as stored in DNA). Hence, there are conceptual connections between generative art and Artificial Life as well as practical ones.

Generative/process-based art is no longer treated as a fresh field for aesthetic exploration. Fascination with its possibilities seemed to fade sometime after its heyday in the late 1960's. This of course was the time of the Cybernetic Serendipity exhibition in London, Jack Burnham's text Beyond Modern Sculpture and much other activity linking art and computer technology in innovative ways. Recently artists involved in Artificial Life have re-trodden some of the ground cleared by their predecessors in cybernetic art, and have cleared some new space for themselves. Now that Artificial Life is also unfashionable, perhaps a serious assessment of its past and future contributions can be made whilst side-stepping the hype which initially accompanied the field.

The first section below begins by discussing the sense of wonder people often feel whilst contemplating nature or simple physical systems. This is then related to the tradition of the sublime in aesthetics. The paper then discusses means of employing Artificial Life techniques to explore the computational sublime such that a computational system emulating the physical world's capability to generate complexity and novelty might be devised. 


\section{Wonder and the sublime in art and nature.}

People stare contemplating the ocean as it swells and crashes on a rocky shore. They gaze fixedly into a fire until the sting of smoke raises their awareness. People may lie on their backs and follow passing clouds, or marvel at the glittering of stars. There are many things which fascinate us, which mesmerize us, which cause us to forget ourselves and our situation as we become lost in a timeless appreciation of nature.

There are also circumstances under which we may have an experience that reminds or forces us to consider our insignificance: the feeling which accompanies the vastness of spaces such as the ocean, a desert plain or an endless mountain range, or the feeling of insignificance when contemplating the age of the universe or the years over which a trickle of water has eroded a canyon for example. These expanses can nevertheless be made subject to reason. We are mentally equipped to discuss the concept of infinity, even if we are unable to quite fathom it intuitively. This paradoxical experience Kant has labelled the mathematical sublime [Feagin, 1995].

Likewise Kant introduces the dynamically sublime as relating to encounters with the ferocity of nature and the sense of vulnerability this entails, coupled with the triumph of reason over fear. In his own writings on the sublime, Edmund Burke takes the view that the sensation of incomprehensibility, the fear of hopelessness or of danger, coupled with the knowledge that one is i able to reason about something beyond one's senses, or one is not inadequate or in danger, causes a kind of delight through internal conflict - a sublime experience [Hibble 1957, pp83-98]. That is to say there is an element of the sublime, perhaps not an artistic element, in resisting the urge to flee as a tiger roars from behind bars. One's body responds with fear to the situation, but reason easily overcomes this and forces the body to stand its ground - generating a sense of delight tinged with terror. In the case of a painting, the viewer's separation from a wild scene of stormy seas or a vast desert is not enforced by iron bars, but by the picture plane. This is coupled with the knowledge "it is only a picture".

How can a work of art, specifically a work of computer-based, generative software, approach the sublime? Whilst there is a vast amount of literature on the sublime in art dating back as far as Longinus [Longinus, 1963], the focus of this paper is a little more narrow. A recent publication [McCormack \& Dorin, 2001] outlines the computational sublime. This arises from viewing the computer in terms of its capability to perform logical operations at a rate and on a scale vastly outside our own abilities in this area. Due to its speed, the computer is able to mediate between our human perceptual apparatus and (practically) infinite computationally-defined spaces. Yet, since we are the makers and programmers of these machines, our power of reason is not only able to overcome, but define these very spaces which our senses are unable to grasp fully.

Before discussing computer-based art in more detail, some much simpler artefacts, possibly just for a moment, rival natural wonders in the fascination they hold. How does this relate to the sublime? It seems that there are a number of methods by which the sensation may be encouraged and that some of these have been recreated in artefacts specifically for this purpose. They are categorized below for convenience.

Marking time: Maybe the simplest examples of our mesmerizing creations include a stream of liquid running from a water-clock or fountain, the shifting sands of the hour-glass and the oscillating pendulum. Each of these marks time in its own manner: one a continuous stream; the next a finely particulate flow; and the last in clear, discrete stages. The eternal flow, the innumerable sand particles, the neverceasing oscillations, confront us with the infinite through this visible marking of time. Although these natural processes were utilized in desk ornaments to amuse the bored 
office worker of the 1980 's, even in "serious" art, simple processes like this may give a sense of the sublime.

Exposing spaces: The wire-suspended mobiles of Calder successfully employ mechanical processes in a manner which has been accepted within the art world [Rower, 1998]. Calder's playful pieces are captivating and elegant for all their simplicity. Their workings are laid plainly before the viewer, all that they are is apparent at a glance - and yet this is not so, for their movement brings a vitality and opens a space which the static sculpture does not possess. The universe a mobile sweeps out is contained within its wires, rods and solid forms, so in one sense they may be held in (and created from) the palm of a hand. Yet as they are touched by invisible air currents their inner complexity is exposed.

Intricacy: Many a visitor has been fascinated by the button-driven clockwork and gearing of exhibits in traditional science museums. Here a sense of wonder at the machine in its entirety arises, but also a fascination with the intricacy of the mechanism. Each gear meshes with another, each component is configured "just so" and together the pistons and wheels turn in harmony to produce a composite which might drive a clock, crush a quartz boulder, pump water or power a vehicle. This is not the beauty of a crashing ocean or a sunset, but the charm of peering into an ant's nest or through a microscope at a drop of pond water. There is something in these systems which causes one to marvel at a complexity that is just beyond grasp.

Defying the natural order: There is still another wonder to be described here, that of somehow defying the natural order of things. It appears wonderful that a huge boulder might come to be balanced on a slender rock column; that a gyroscopic top remains on its tip despite interference; that a bird or a massive aeroplane can remain suspended in the air, or a colossal steel ship can float; that a magnet can push another away without touching it. These things of course are dealt with to some extent by simple science. Sadly, only children may wonder at these things. Yet implicitly these interactions remain in need of continual re-explanation since each instils apprehension through not being quite "right". That boulder or spinning top might topple at any moment. That bird should fall from the sky and the ship ought to take on water. The magnets are behaving unnaturally. All of these systems cause one to ponder, "How can this be?", even if one can reason about the answer through science.

Curiosity: Related to all of the above phenomena, in particular the previous one, attention can also be held by riddles and intellectual pursuits. Included in this are mind games such as chess, paradoxes, and mathematical puzzles, but also scientific enquiry. These all captivate through our drive to learn why something behaves as it does, especially if it seems to defy the natural order of things. Of the algorithmic art exhibition at Xerox PARC in 1994, Bern writes, "The appearance of algorithmic visual art should raise the temporal questions of "How is this made?" and "Can this be extended?"'" [Bern, 1999].

Whilst the use of the term should, is of questionable accuracy, nevertheless there is something of relevance in the idea that algorithmic art may invite a question and arouse curiosity as much as it satisfies visual aesthetic criteria associated with traditional media.

Any of the devices dealt with above (and no doubt many besides) may be used to convey a sense of the sublime. All of them expose us to our own limitations in terms of comprehension, experience or endurance, whilst simultaneously presenting us with the power to reason about or to encapsulate a phenomenon in a word or representation, or the opportunity to walk away unharmed. Of course the context in which the occurrences and objects above are encountered will have much to say about the extent 
to which the sensation derived relates to art. However the potential to employ any of these basic devices for artistic purposes in search of the sublime experience is present.

\section{Sublime software.}

Now we return to the issue at hand - writing Artificial Life software for artistic purposes. There are of course infinitely many reasons why an artist might wish to do this. Let us suppose in this instance that the goal was software which surprised, not only a viewer, but the artist who fashioned the work. This is in keeping with a proposal made elsewhere by the author [Dorin 2004]. The surprise required might not be a trivial one-off shock, but a genuine and continuing fascination with the novelty of the outcome which is generated, combined with a sense of being lost in a complex world beyond one's grasp. The work requires an aesthetic quality which exhibits the character listed above as intricacy and also at the level listed as curiosity.

It so happens of course, that the techniques of Artificial Life are well suited to this application, Conway's Game of Life cellular automata (CA) being a case in point [Gardener, 1970]. CA's are fascinating for their ability to (practically, if not theoretically) produce ever-fresh patterns. Like clockwork automata or Calder's mobiles, the patterns produced may fall well within a limited domain, often even a cycle. However, especially in a large-scale configuration, there is always some aspect of the system which remains to be discovered in the vast universe these interactions define.

The innovative design pair, the Eames, understood this aspect of a complex visual field. They used their understanding effectively in the 1964 New York, World Fair exhibit for IBM to build a multi-screen cinema. The screens displayed related material simultaneously in such a way as to make it impossible to view all of the footage in detail. Viewers could watch the spectacle as a whole and see it as a multi-screen montage, or they could pick and choose elements to observe in detail - much as one examines a complex clockwork machine, a microscopic world or a beehive piecemeal.

On a related topic, Tufte indicates the benefits for presenting information in parts grouped carefully together, "Panorama, vista and prospect deliver to viewers the freedom of choice that derives from an overview, a capacity to compare and sort through detail" [Tufte, 1990, p.38]. Such considerations also assist painters in creating worlds which reward careful study of their detail. For example, Hieronymous Bosch's Garden of Delights (c. 1500) portrays a world over which the eye may wander at will as it takes in relationships between couples, groups of people, sections of garden and so on. Of course where the object under study is static, a viewer may take the time to examine and re-examine the various parts of a display and avoid missing anything of importance (as Tufte would prefer). When the object is constantly changing, as with multi-screen cinema or a Game of Life run, an investigation of one element results in irrevocably missed information about another. Under these circumstances, no matter where a viewer's attention is focussed, they are left with the impression that an abundance of chances were missed. Their faculties are fundamentally unable to absorb all that is before them.

Whilst one of the attractions of the Game of Life occurs at the visual level, the software is also an attractive subject for philosophical discussions concerning emergence and complex systems [Dennett, 1991]. It contains a vast number of possibilities within the bounds of its simple virtual-physics. Whilst its transition rules may be considered in terms of biological analogy (over-crowding and mutual support of living cells for instance), the result is still an intrinsically digital system. Cells are in 
one of exactly two states and their behaviour is completely deterministic. Yet from this emerges a milieu of flickering forms which somehow suggest life, without mimicking it.

The question "How does it work?" is implied by any given run of the Game of Life in which it is recognized that the output is not at all random, but highly organized and structured according to its own internal rules. Even once these rules are known to the viewer, that they are capable of creating such a bewildering outcome remains a source of fascination to the Artificial Life researcher and to the newly-informed student alike.

\section{The betrayal of points and lines.}

Having briefly discussed the concepts of wonder and the sublime, this section now addresses the representational schemes which may be used to bring the digital realm to the realm of sublime experience. Of particular interest is the way in which points of light may be used to give the bounded computer screen the appearance of intricacy and an ability to expose unbounded space (see the earlier categorization of methods for approaching the sublime). This has already been touched upon whilst discussing the Game of Life in the previous section.

Taking the lead of Kandinsky, the discussion begins with the geometric point, an invisible thing too perfect to exist [Kandinsky, 1979]. An artist may approximate it by instigating a collision between a sharp tool and a flat plane. The artist's point has character. It is not one of Plato's Ideal Forms but it is beautiful for all its imperfection.

The point may also be displayed on a CRT or LCD monitor. The size and shape of the smallest displayable shape are dictated by the display technology. Beyond a single pixel the character of the point may also be altered, albeit with less potential for variation than in the analogue world.

Anyone familiar with Islamic or Roman mosaics, medieval tapestry, Pointillist painting, the television screen or a computer monitor, is aware that in vast numbers, the point may vanish in an ocean of its kind. Here it becomes simply one atom in a larger texture - mobs of points are not as honest as individuals.

If the draftsman drags a pen across the page another form results, a line. Like the point, the line seems unpretentious, especially if it is straight and completely surrounded by white space. However, place a few straight lines together and important transformations may take place. For example, the triangle is born of three lines and is eventful for its ability to enclose space on the plane and thereby define a boundary, and with it, an object. The second transformation of relevance here is the apparent shift into three dimensions brought about by the Cartesian axes. This is also born of three lines and is eventful for its ability to suggest space which extends beyond the plane in which the lines are drawn.

Under some circumstances the three lines which represent Cartesian space may appear simple and flat, under others however, the third dimension "pops" out of the plane at the viewer. It is outside the scope of this paper to discuss how this second transformation occurs, the interested reader is referred to [Hoffman, 2000]. For now it is sufficient if it be acknowledged that at least in some cases, three Cartesian axes suggest a volume where there is none, this being (of course) the principle which underlies Renaissance painting and a host of styles up to and, of relevance to this discussion, including modern computer graphics. Using only lines one may build a twodimensional representation of a solid object in unbounded three-dimensional space.

To return at this stage to the Game of Life, the system is typically displayed as, strangely enough, a grid of points. The choice of the point as the basic element of the simulation excludes the misleading suggestions of line drawings. Still, even within this limited visual space, the CA is successful at its evocation of life and with it intricacy. 
How can similar systems expose or suggest spaces which are infinitely large and intricate?

\section{Moving beyond two dimensions.}

The Game of Life and CA-based generative art such as IMA Traveller [Driessens \& Verstappen, 2001] work in the domain of points and suggestion, as outlined above. With a computer monitor it is easy to represent lines and surfaces using a multitude of similarly lit pixels.

IMA Traveller, whilst working with points in a plane, recursively sub-divides these, giving the viewer the sensation of a bottomless plummet. This is an interesting perceptual phenomenon when it is considered that by continuously zooming in on a two dimensional image (the points aren't modelled in three dimensional space) one can instil the sensation of motion through a continuous and infinitely detailed universe. The fractal zooms of the 80's are perhaps the most familiar and overused form of this same trick. In their work Powers of Ten (1968), the Eames also utilized rapid scale shifts to produce a film of great effect.

The CA and its artistic derivatives draw attention for evoking natural phenomena, and for prompting the viewer to determine the underlying principles by which they operate. Not all Artificial Life software is of this type, even if it is important to the research field for other reasons. One may propose a full range of works of which the CA-based software falls near the centre. This spectrum runs from abstract systems such as Ray's Tierra [Ray, 1991], to clearly representational systems such as Sims' Virtual Creatures [Sims, 1994].

Ray's work emphasizes the principles of selection and evolution through rapid reproduction, and has itself proved a useful model and a starting point for other researchers such as Pargellis [Pargellis, 2001] (discussed below) to further the study of evolutionary systems. However Ray's work does not have the visceral appeal which visual cellular automata may have. Tierra's space of machine code instructions is abstract and not typically represented in such a way as to be comprehended by the eye. A fascination with Tierra arises through careful study and is predicated upon an understanding of the core workings of the system and the way in which the instructions interact and occupy memory. This contrasts with the CA in which the rules need not be known to understand the system at one level. It is this first level of visceral comprehension which prompts theorizing about what underlies the behaviour of the CA cells.

At the opposite end of the spectrum, Sims' Virtual Creatures fascinate researcher and layperson alike. His jumping, swimming, running and limping creations are so full of character that one easily takes a leap of faith by referring to them as "creatures", even though their bodies are clearly rendered cuboids. This leap erases any chance of a viewer posing the question "How does it work?" because it is implied by the visualization that these are creatures! Whilst a graphics researcher or software engineer may see Sims' video and wonder at the means of producing such marvellous results, this question is not implied by the creatures' visualization as it is by the CA. The virtual creatures do not operate according to unknown rules, we are tricked into believing that they operate according to the rules all creatures obey. There is seemingly nothing here to discover.

This aspect of Sims' creatures is largely due to the way in which they are visualized - the representations of three dimensional space, of solids and their surfaces, of friction and other forces are all customary. In this case the visualization is prescriptive, rather than evocative. McCormack's Eden, which was constructed as an artwork (see article in this issue and [McCormack, 2001]), similarly represents 
organisms and their real-world behaviours. But it displays them using iconic forms on a two-dimensional grid (mapped to an " $X$ " in three dimensional space) and in a world nevertheless governed by rules of survival, energy, mating and space, based on those of the real-world. In contrast with Sims' work, this underlying link to the physical world is not prescribed and takes some experience to decipher... if a viewer is able to decipher it at all. Although the system is still considerably more representational than the Game of Life, what remains is an aesthetic visual experience akin to viewing an intricate CA, coupled with the implicit question, "How does it work?"

This discussion of Ray's, Sims' and McCormack's work is in no way intended as an evaluation of their worth. These examples only serve to illustrate the various ways in which a viewer may contemplate a generative process and its outcome, and therefore the various ways in which the devices may be employed for artistic purposes. The next section takes current generative artworks and Artificial Life software as a starting point. It explores areas into which artists might move to further expand the sense of wonder their works inspire.

\section{Spaces that build themselves.}

An artist aiming to produce a system capable of sustaining a continuous increase in complexity shares a goal with many Artificial Life researchers [Bedau et al, 2000]. Why would an artist wish to do such a thing? To return to the ideas discussed above, the artist searching for the computational sublime would find it, perhaps in its ultimate form, by generating an experience of open-ended complexity governed by processes instantiated on a computer. The loss of control would be complete - the system would build its own structures and define its own universe. It would do this according to human-engineered rules, yet in a way which defies humans to anticipate its outcome.

This system would be following the code a programmer laid down and run on a machine an engineer designed. Would this device behave in a way about which we could easily reason? In this conflict between control and riot lies the sublime. Mary Shelley knew this well, her friends and contemporaries were much interested in the sublime, when she vividly penned Frankenstein and his monster run amok [Shelley, 1989]. For a historical overview of theories of the sublime in this period see [Hipple, 1957].

How does one write code which will produce the hierarchically-organized composite structures associated with life and ever increasing complexity? If we consider the cells of a CA grid as analogous to molecules, and higher level emergent structures such as gliders as analogous to organelles (a far stretch when one considers the complexity of interactions a physical molecule or an organelle may undergo, and the feeble interactions between neighbouring cellular automata), can we code the system so that still larger-scale groupings of structure occur? Can it produce structures at the level of single cells, a multi-cellular organism or an ecosystem?

In theory, even gliders, spinners and other structures of the Game of Life may be carefully arranged into larger scale units (such as a self-reproducing machine incorporating a universal Turing machine, if one has the patience to arrange its $10^{13}$ cells [Dennett, 1991, p109]). The question remains though, is it possible that such higher level structure will appear of its own accord? If software could be arranged to facilitate this, the structures which arose would do so on their own terms and might therefore behave in ways not envisaged by the creator. This might provide an effective source of complexity to assist an artist in their search for the computational sublime.

If theories about the process of natural increase in complexity (such as those extensively discussed by Kauffman for example [Kauffman, 1993]) hold true in virtual systems, then the elements in the virtual space might self-assemble into simple stable 
structures. Perhaps, if the virtual physics and chemistry of the world allowed it, simple reactions might occur, possibly some in auto- and cross-catalytic sets such as those described by Dorin [Dorin, 2000]. These might form the basis of a recognizable topology with the bare bones of a metabolism. What next?

As natural evolution demonstrates, one way to achieve an increase in complexity beyond this is to have the structures engage in a reproductive battle against one another for resources (see [Taylor, 2002]). Pargellis' system Amoeba manages to initiate reproduction randomly. It establishes conditions in which practical CPU and memory resources are sufficient for a replicator to appear spontaneously from the pre-biotic soup [Pargellis, 2001]. In a Tierra run such an event is much less likely than in Amoeba. Hence Ray initially seeded Tierra's population with a replicator to allow evolution to commence. The problem of coding a simulation which can make the leap from self-organization to spontaneous evolution of structure seems (as far as this author is aware) unmade by any researcher.

Setting aside the leap from self-organization to evolution, even though systems solely employing artificial evolution are readily implemented, getting these to mimic natural evolution's progression from molecules to organelles to cells and on to multicellular creatures and ecosystems, has proved a stumbling block. Although worlds such as SOCA give rise to auto- and cross-catalytic sets, Amoeba may randomly give rise to replicators, and Polyworld [Yaeger, 1994] gives rise to simple communities, there has been limited success (arguably no success) in writing software which encompasses more than one of these important level-shifts without resorting to abstractions so high that the simulations they are contained within become trivial.

The reasons for this difficulty are not yet clear. In part, current computational resources may be to blame. However this is quite likely only a part of the story, and maybe only a small part at that. Rasmussen for instance, has suggested that the bottom level of our simulations are not complex enough to give rise to the kind of multilayered outcomes we desire. He proposes that only by adding complexity to the bottom-level elements of a simulation can we expect to gain extra levels of organized structure on top of any earlier ones [Rasmussen et al, 2001]. This claim seems to contradict the "complex systems dogma" which explicitly treats complex phenomena as emergent from simple interactions. Since in Rasmussen's paper notions of "complexity" and "adding complexity" are only loosely defined, it is not clear exactly how and to what extent this might be the case. This is discussed in detail elsewhere [Dorin \& McCormack 2002].

Rasmussen supports his view with a claim about a model that he has constructed. That this model does not in fact demonstrate the emergence of multiple levels, and that a similar outcome can be obtained without adding complexity at the base layer, is argued by Gross and McMullin [Gross \& McMullin, 2001].

It is outside the scope of this paper to become too deeply embroiled in this battle. Rasmussen's suggestion may in some sense prove true. Either way, further questions need to be addressed simultaneously. Might there be a limit to all this "complexity adding"? How much added complexity is necessary to move from one level to the next? Questions like these remain open and continue to be debated.

Besides issues raised above, there may be fundamental problems with current approaches to solving the problem. It is possible that simulations on current computer architectures and employing computer programs as they are currently understood, will turn out to be practically limited in their ability to produce the kind of truly open-ended complexity increase required. Issues of available resource consumption are an obvious reason why infinite increase is impossible, however are there reasons why any interesting string of increases in complexity may be impossible with current 
programming and computer technology? These questions remain a topic for a further paper and, in one sense (counter to the claims of the Renaissance writer Vasari about artists surpassing nature [Vasari, 1976]), the hope of one day creating a virtual space as multi-faceted as nature remains faint.

\section{Conclusion}

Although the limitations of our abilities to code multiple-level hierarchies are apparent, clearly this does not imply that our art is similarly constrained. The element of the sublime in a Caspar David Friedrich canvas does not arise from the intricacy of its mechanism, but from contemplating nature and our place in it from behind the safety of a picture plane. Even more apparent is the irrelevance of intricacy and nature (taken literally) to postmodern interpretations of the sublime such as those discussed by Jean-Francois Lyotard [van de Vall, 1995]. Hence works such as the dark canvasses produced in Mark Rothko's later years may be discussed in terms of their contribution to the postmodern sublime. The sublime does not lie in a work, rather the work may act to trigger a sublime experience in a viewer. In the case of Lyotard's ideas, this relates to a sense of formlessness and therefore of things which may be better left un-presented.

Since our machines are faster at mathematics than ourselves they will always maintain the ability to play the role of mediator between us and the vast computational spaces outside our direct experience. Perhaps one day these same machines will be able to take us beyond spaces which look more and more familiar as we travel through them, into spaces which increase in complexity and continue to surprise us. Here the sublime experience of nature's vastness and ferocity may be rivalled through a sense of the computational sublime. We will be sensing a space rendered maybe with points on a plane and computed on-the-fly by our fastest machines, and it will seem to us as terrible and delightful as standing on an icy summit surveying all the world.

\section{References}

Bedau, M.A., McCaskill, J.S., Packard, N.H., Rasmussen, S., Adami, C., Green, D.G., Harvey, I., Ikegami, T., Kaneko, K., Ray, T.S., 2000, “Open Problems in Artificial Life”, Artificial Life 6, Bedau \& Taylor (eds), MIT Press, pp363-376

Bern, M., 1999, “Art Shows at PARC” in Art and Innovation, Harris (ed.), The MIT Press, pp259-277

Brown, P, 1999, "Sandlines", in Process Philosophies, Dorin \& McCormack (curators) described in Brown, P., "Stepping stones in the mist", in First Iteration: Proceedings of the first international conference on generative systems in the electronic arts, CEMA, Dorin \& McCormack (eds.) Melbourne, Australia

Dennett, D.C., 1991, "Real Patterns”, Journal of Philosophy, 88, pp27-51

Dorin, A., 2000, "Creating a Physically-based, Virtual-Metabolism with Solid Cellular Automata", in Proceedings Artificial Life 7, Bedau et al (eds), MIT Press, pp13-20

Dorin, A., McCormack, J., 2002, "Self-Assembling Dynamical Hierarchies", in Proceedings of Artificial Life 8, Standish et al (eds), MIT Press, pp423-428

Dorin, A., 2004, "The Virtual Ecosystem as Generative Electronic Art", in Proceedings of 2nd European Workshop on Evolutionary Music and Art, Applications of Evolutionary Computing: EvoWorkshops 2004, Coimbra, Portugal, April 5-7, Günther R. Raidl, Stefano Cagnoni, Jürgen Branke, et al (eds), Springer-Verlag Heidelberg, pp467-47

Driessens, E., \& Verstappen, M., 2001, keynote presentation in Proceedings of Second Iteration, second international conference on generative systems in the electronic arts, Dorin (ed.), CEMA, Melbourne, Australia, pp12-13

Feagin, S.L., 1995, "Sublime”, in The Cambridge Dictionary of Philosophy, Audi (ed.), Cambridge University Press, p774

Gardner, M., 1970, “The Fantastic Combinations of John Conway's New Solitaire Game, 'Life”, Scientific American 223(4), pp120-123 
Gross, D., McMullin, B., 2001, “Is it the Right Ansatz?, Artificial Life 7, Bedau \& Taylor (eds), MIT Press, pp355365

Hipple, W.J., 1957, "The Beautiful, the Sublime, and the Picturesque in Eighteenth-Century British Aesthetic Theory", Southern Illinois University Press

Hoffman, D.D., 2000 "Visual Intelligence: How We Create What We See”, Norton

Kandinsky, W., 1979 "Point and Line to Plane”, Dover Publications, New York, (first published 1926)

Kauffman, S.A., 1993, “The Origins of Order”, Oxford University Press

Longinus, 1963, "On the Sublime”, translated by Havell, in Aristotle's Poetics, Demetritus on Style, Longinus on the Sublime, Everyman's Library, Dutton New York, pp.133-202 (first written c. 250 A.D.)

McCormack, J., 2001, "Eden: An Evolutionary Sonic Ecosystem", in Advances in Artificial Life, $6^{\text {th }}$ European Conference, Kelemen \& Sosik (eds), ECAL, Springer, LNAI 2159, pp.133-142

McCormack, J., Dorin, A., 2001, “Art, Emergence, and the Computational Sublime” in Proceedings of Second Iteration, second international conference on generative systems in the electronic arts, Dorin (ed.), CEMA, Melbourne, Australia, pp. 67-81

Pargellis, A.N., 2001, "Digital Life Behaviour in the Amoeba World”, Artificial Life 7, Bedau \& Taylor (eds), MIT Press, pp63-75

Rasmussen, S., Baas, N.A., Mayer, B., Nilsson, M., Olesen, M.W., 2001, “Ansatz for Dynamical Hierarchies”, Artificial Life 7, Bedau \& Taylor (eds), MIT Press, pp.329-353

Ray, T.S., 1991, “An Approach to the Synthesis of Life”, in Artificial Life II, Langton, Taylor, Farmer, Rasmussen (eds), Addison-Wesley, pp.371-408

Rower, A.S.C, 1998, “Calder Sculpture”, National Gallery of Art, Washington, Universe

Shelley, M., 1989, "Frankenstein, the Modern Prometheus”, first published 1818, reprint, Joseph, M.K. (ed.), Oxford Univeristy Press

Sims, K., 1994, "Evolving Virtual Creatures”, in Proceedings of SIGRRAPH 1994, ACM Press, July, pp15-34

Taylor, T., 2002, “Creativity in Evolution: Individuals, Interactions and Environments”, in Creative Evolutionary Systems, Bentley \& Corne (eds), Academic Press, pp. 79-108

Tufte, E.R., 1990, "Envisioning Information", Graphics Press

van de Vall, R., 1995, "Silent Visions, Lyotard on the Sublime", in The Contemporary Sublime, Sensibilities of Transcendence and Shock, Hodges (ed.), Art \& Design, VCH Publishers, pp69-75

Vasari, G., 1976, “The Lives of the Artists”, first published 1568, translated by Bull, G., reprint Penguin Books

Yaeger, L., 1994, “Computational Genetics, Physiology, Metabolism, Neural Systems, Learning, Vision and Behavior or Polyworld: Life in a New Context”, in Proceedings, Artificial Life III, SFI Studies in the Sciences of Complexity, Langton (ed), Addison-Wesley, pp263-298 